\title{
Autorenverzeichnis
}

\section{- Geleitwort}

Univ.-Prof. Dipl.-Ing. Dr. med. (habil.)

Hartmut Witte

Technische Universität Ilmenau

Fakultät für Maschinenbau, Fachgebiet Bio-

mechatronik

D-98684 Ilmenau

Tel.: +49 3677-692460

E-Mail: biomechatronik@tu-ilmenau.de

\section{- Kapitel 1}

Dipl.-Ing. Johannes Dehm

VDE Verband der Elektrotechnik Elektronik

Informationstechnik e. V.

Stresemannallee 15

60596 Frankfurt am Main

Tel.: +49696308-0

\section{Dr. Franz Bartmann}

Vorstand der Bundesärztekammer (bis 09/18)

Liliencronweg 10

24939 Flensburg

\section{- Kapitel 2}

PD Dr. med. Bernhard Clasbrummel

Orthopädische Praxis Clasbrummel

Königstraße 70

70173 Stuttgart

Telefon: +49 711-9979943

E-Mail: praxis@dr-clasbrummel.de

Dipl.-Ing. Torsten Eggers

HELLA Fahrzeugkomponenten $\mathrm{GmbH}$

Dortmunder Straße 5

28199 Bremen

Tel.: +49 $4215951-0$

Prof. Dr.-Ing. habil. Günther Pfeifer

Technische Universität Dresden

Institut für Halbleiter- und Mikrosystemtechnik

Helmholtzstr. 10

01062 Dresden apl. Prof. Dr.-Ing. habil. Uwe Marschner

Technische Universität Dresden

Institut für Halbleiter- und Mikrosystemtechnik

Helmholtzstr. 10

01062 Dresden

Tel.: +4935146335399

E-Mail: uwe.marschner@tu-dresden.de

Prof. Dr.-Ing. habil. Roland Werthschützky

Technische Universität Darmstadt, S3/06 136

Merckstraße 25

64283 Darmstadt

E-Mail: werthschuetzky@must.tu-darmstadt.de

\section{Dipl.-Ing. Michael Görtz}

Fraunhofer Institut für Mikroelektronische Schaltungen und Systeme

Finkenstr. 61

47057 Duisburg

Tel.: +49 2033783122

E-Mail: michael.goertz@ims.fraunhofer.de

Prof. Dr. rer. nat. Wilfried Mokwa

Rheinisch-Westfälische Technische Hochschule

Aachen

Institut für Werkstoffe der Elektrotechnik 1

Sommerfeldstraße 24

52074 Aachen

Tel.: +49 $24180-27810 / 1$

E-Mail: mokwa@iwe1.rwth-aachen.de

Prof. Dr.-Ing. Hoc Khiem Trieu

Technische Universität Hamburg

Institut für Mikrosystemtechnik

Eißendorfer Straße 42 (M)

21073 Hamburg

Tel.: +4940428784398

E-Mail: trieu@tuhh.de

Prof. Dr.-Ing. Uwe Schnakenberg

Rheinisch-Westfälische Technische Hochschule

Aachen

Institut für Werkstoffe der Elektrotechnik 1

Sommerfeldstraße 24

52074 Aachen

Tel.: +49 241 80-27842

E-Mail: schnakenberg@iwe1.rwth-aachen.de 
Prof. Dr. Thomas Stieglitz

Lehrstuhl für Biomedizinische Mikrotechnik, Institut für Mikrosystemtechnik (IMTEK),

BrainLinks-BrainTools

(Exzellenzcluster DFG EXC 1086)

Albert-Ludwigs-Universität Freiburg

Georges-Köhler-Allee 102

79110 Freiburg

Tel.: +497612037471

E-Mail: stieglitz@imtek.uni-freiburg.de,url: http://www.imtek.de/bmt

Prof. Dr. med. Dr. h. c. Thomas Zahnert

Klinikdirektor, Klinik und Poliklinik für Hals-, Nasen- und Ohrenheilkunde

Universitätsklinikum Carl Gustav Carus

Fetscherstr. 74

01307 Dresden

Tel.: +49 3514584420

E-Mail: orl@uniklinikum-dresden.de

Dr.-Ing. Martin Pachen

Hella GmbH \& Co. KGaA

Rixbecker Str. 75

59552 Lippstadt

E-Mail: Martin.Pachen@hella.com

Prof. Dr. med. Omid Majdani

Chefarzt, Klinikum Wolfsburg, Klinik für Hals-, Nasen-, Ohrenheilkunde, Kopf- und Halschirurgie

Sauerbruchstr. 7

38440 Wolfsburg

Tel.: +49 $536180-1961$

Dipl.-Ing. Renè Körbitz

Technische Universität Dresden

Institut für Halbleiter- und Mikrosystemtechnik

Helmholtzstr. 10

01062 Dresden

E-Mail: rene.koerbitz@tu-dresden.de

Dr.-Ing. Holger Neubert

Fraunhofer-Institut für Keramische

Technologien und Systeme IKTS

Intelligente Materialien und Systeme |

Smart Materials and Systems

Winterbergstraße 28

01277 Dresden

Tel.: + $493512553-7615$

E-Mail: holger.neubert@ikts.fraunhofer.de
Prof. Dr. rer. hum. biol. Felix Capanni

Technische Hochschule Ulm

Forschungsgruppe Biomechatronik

Albert-Einstein-Allee 55

$89081 \mathrm{Ulm}$

Tel.: +49 731 50-28521

E-Mail: felix.capanni@thu.de

Prof. Dr. rer. nat. Bernhard Wolf

Technische Universität München

Arcisstr. 21

80333 München

Leitung Steinbeis-Transferzentrum

Medizinische Elektronik und

Lab on Chip-Systeme

Fendstr. 7

80802 München

Dr.-Ing. Helmut Grothe

Akademischer Direktor

Fakultät Elektrotechnik und Informationstechnik

Technische Universität München

Arcisstr. 21

80333 München

Dr. Johannes Clauss

AdjuCor GmbH

Neumarkter Str. 18

81673 München

\section{M.Sc. Nicolas Bader}

Technische Hochschule UIm

Forschungsgruppe Biomechatronik

Albert-Einstein-Allee 55

$89081 \mathrm{Ulm}$

E-Mail: nicolas.bader@thu.de

Cand. Dr. med. Dipl.-Ing. (FH)

\section{Christian Peschmann}

Anästhesie

Sana Klinik Biberach

Ziegelhausstraße 50

88400 Biberach an der Riß

E-Mail: christian.peschmann@gmail.com

Prof. Dr. med. Marc-Eric Halatsch

Leitender Oberarzt, Klinik für Neurochirurgie

Universitätsklinikum Ulm

Albert-Einstein-Allee 23

$89081 \mathrm{Ulm}$ 
Dr. med. Jürgen Straube

Innere Medizin und Kardiologie

Schulweg 5

01640 Coswig

E-Mail: juergen.straube@gmx.net

Univ.-Prof. Dr. med. Philipp Sommer

FHRS, FESC, FEHRA

Universitätsklinik der Ruhr-Universität Bochum

Klinikdirektor, Klinik für Elektrophysiologie und

Rhythmologie

Herz- und Diabeteszentrum NRW

Georgstr. 11

32545 Bad Oeynhausen

E-Mail:psommer@hdz-nrw.de

Dr. rer. nat. Dirk Frömer

Training \& Education CRM CENEMEA

BIOTRONIK Vertriebs GmbH \& Co. KG

Woermannkehre 1

12359 Berlin

E-Mail: dirk.froemer@biotronik.com

Dipl.-Ing. Karl-Heinz Otto

tricumed Medizintechnik GmbH

Röntgenstraße 7a

24143 Kiel

Tel.: +49431709900

E-Mail: karl-heinz.otto@tricumed.de

\section{Thomas Otto}

Zum Exerzierhaus 9

14469 Potsdam

E-Mail: Thomas.Otto.Medizin@web.de

Prof. Dr.-Ing. Andreas Richter

Technische Universität Dresden

Institut für Halbleiter- und Mikrosystemtechnik

Helmholtzstr. 10

01062 Dresden

E-Mail: andreas.richter7@tu-dresden.de

\section{Dipl.-Ing. Martin Schmidt}

Technische Universität Dresden

Fakultät Elektrotechnik und Informationstechnik Institut für Biomedizinische Technik

Fetscherstr. 29

01307 Dresden

E-Mail: martin_schmidt@tu-dresden.de
Prof. Dr.-Ing. habil. Hagen Malberg

Technische Universität Dresden

Fakultät Elektrotechnik und Informationstechnik Institut für Biomedizinische Technik

Fetscherstr. 29

01307 Dresden

E-Mail: hagen.malberg@tu-dresden.de

Prof. Dr. sc. techn. habil.

Dipl. Betriebswissenschaften Frank Ellinger

Technische Universität Dresden

Fakultät Elektrotechnik und Informationstechnik

Professur für Schaltungstechnik und

Netzwerktheorie

Helmholtzstr. 10

01062 Dresden

E-Mail: frank.ellinger@tu-dresden.de

Prof. Dr.-Ing. Dirk Plettemeier

Technische Universität Dresden

Fakultät Elektrotechnik und Informationstechnik

Professur für Hochfrequenztechnik

Helmholtzstr. 10

01062 Dresden

E-Mail: dirk.plettemeier@tu-dresden.de

- Kapitel 3

Prof. Dr. med. Dipl.-Inform. Georgios Raptis

Ostbayerische Technische Hochschule

Regensburg

Fakultät Informatik und Mathematik

Prüfeninger Str. 58

93049 Regensburg

E-Mail: georgios.raptis@oth-regensburg.de

Dr.-Ing. Nicolás Gay

Luxoft GmbH

Balanstraße 73

81541 München

E-Mail:ngay@luxoft.com

- Kapitel 4

Dr.-Ing. Hans Günter Despang

Dresdner Str. 100

01456 Langebrück 
Dipl.-Ing. Steffen Netz

Fraunhofer-Institut für Photonische

Mikrosysteme IPMS

Maria-Reiche-Str. 2

01109 Dresden

Tel.: +49 $3518823-0$

Dr.-Ing. Sebastian Sauer

Technische Universität Dresden

Institut für Halbleiter- und Mikrosystemtechnik

Helmholtzstr. 10

01062 Dresden

Tel.: +4935146334939

E-Mail: sebastian.sauer@tu-dresden.de

- Kapitel 5

PD Dr.-Ing. Jürgen Uhlemann

Hellendorfer Strasse 12

01279 Dresden

\section{Dr.-Ing. Sabine Friedrich}

First Sensor Microelectronic Packaging

Grenzstraße 22

01109 Dresden

- Kapitel 6

Dipl.-Ing. Bernhard Kleiner

Melexis Dresden $\mathrm{GmbH}$

Zur Wetterwarte 50

01109 Dresden

Dipl.-Ing. Tom Gothan

Technische Universität Dresden

Fakultät Verkehrswissenschaften „Friedrich List“

Institut für Automobiltechnik Dresden (IAD)

George-Bähr-Str. 1b

01069 Dresden

Tel.: + 49 351-463-38614

Email: tom.gothan@tu-dresden.de

\section{- Kapitel 7}

Dr. rer. nat. Birger Jettkant

Ruhr-Universität Bochum

Institut für Prävention und Arbeitsmedizin der Deutschen Gesetzlichen Unfallversicherung (IPA)

Bürkle-de-la-Camp-Platz 1

44789 Bochum

E-Mail: Jettkant@ipa-dguv.de

\section{- Kapitel 8}

Dr.-Ing. Andreas Heinig

Fraunhofer-Institut für

Photonische Mikrosysteme IPMS

Maria-Reiche-Str. 2

01109 Dresden

E-Mail: andreas.heinig@ipms.fraunhofer.de

Prof. Dr.-Ing. habil. Wolf-Joachim Fischer

Technische Universität Dresden

Institut für Halbleiter- und Mikrosystemtechnik

Helmholtzstr. 10

01062 Dresden

E-Mail: wolf-joachim.fischer@tu-dresden.de

\section{- Kapitel 9}

Dr.-Ing. Dipl.-Wirt.-Ing. Peter Dültgen

FGW Forschungsgemeinschaft Werkzeuge

und Werkstoffe e. V.

Papenberger Straße 49

42859 Remscheid

Tel.: +49 2191900300

E-Mail: dueltgen@fgw.de

Dr. rer. medic. Christoph Fischer

Roche Diabetes Care GmbH

Sandhofer Straße 116

68305 Mannheim

E-Mail: christoph.fischer@ieee.org

Dr.-Ing. Gerd Gottlebe

Bannewitzer Str. 52

01705 Freital

Tel.: +493514965376

E-Mail: post@gottlebe.ch

\section{- Kapitel 10}

\section{Dr. Anna-Maria von Saucken}

Hochschule für Technik und Wirtschaft Berlin Fachbereich 4: Informatik, Kommunikation und Wirtschaft, Angewandte Informatik (B) momedica ${ }^{\circledR} \mathrm{GmbH}$, Medical Technology and Mobile Services

Petersburgerstr. 61

10249 Berlin

Tel.: +49 $3024131-0$ 
M. Sc. Anna Zirk

Technische Universität Berlin

Institut für Psychologie und Arbeitswissenschaft

Marchstr. 23

10587 Berlin

\section{M.Sc. Bettina Seifert}

Technische Universität Berlin

Institut für Psychologie und Arbeitswissenschaft

Marchstr. 23

10587 Berlin 
